Supporting Information

\title{
Effect of polymer binders with single-walled carbon nanotubes on the electrochemical and physicochemical properties of the $\mathrm{LiFePO}_{4}$ cathode
}

\author{
Aleksei V. Kubarkov,*, Artem A. Asharchuk ${ }^{\mathrm{a}}$, Oleg A. Drozhzhin ${ }^{\mathrm{a}, \mathrm{b}}$, Evgeny A. Karpushkin, \\ Keith J. Stevenson ${ }^{\mathrm{a}, \mathrm{b}}$, Evgeny V. Antipova, ${ }^{\mathrm{a}, \mathrm{b}}$ Vladimir G. Sergeyeva,*
}

${ }^{a}$ Department of Chemistry, Lomonosov Moscow State University, Leninskie Gory 1-3, 119991

Moscow, Russia;

${ }^{\mathrm{b}}$ Skolkovo Institute of Science and Technology, Nobel Street 3, 121205 Moscow, Russia.

Corresponding authors:

*Aleksei V. Kubarkov. E-mail: aleksei.kubarkov@gmail.com

*Vladimir G. Sergeyev. E-mail: sergeyev@ genebee.msu.ru

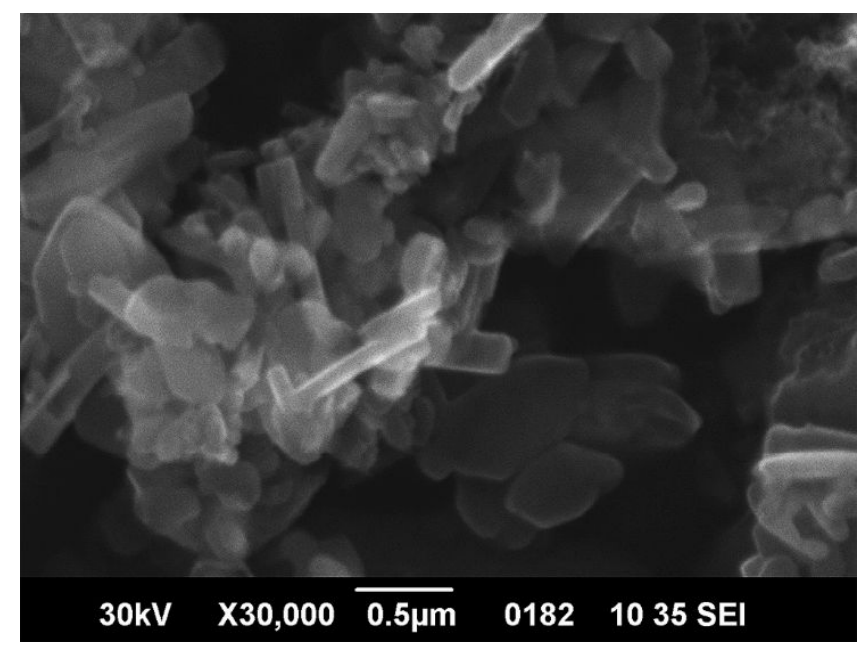

Figure S1. SEM-image of the LFP material. 


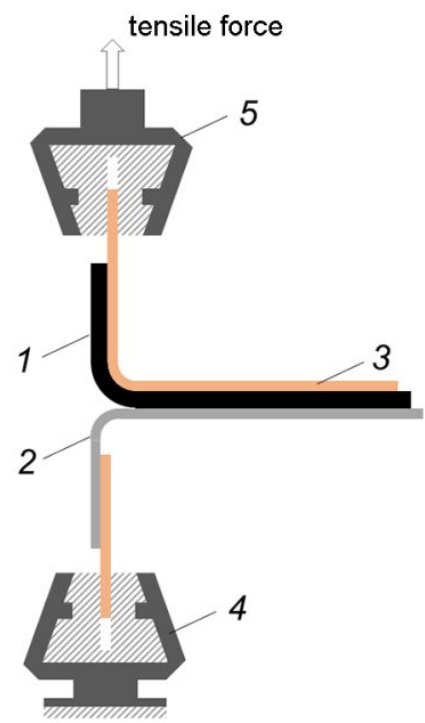

Figure S2. Scheme of the measuring the coating peeling force of the composite electrode films; 1 - composite cathode, 2 - Al foil (current collector), 3 - adhesive tape, 4 - fixed clamp, 5 - movable clamp.

(a)

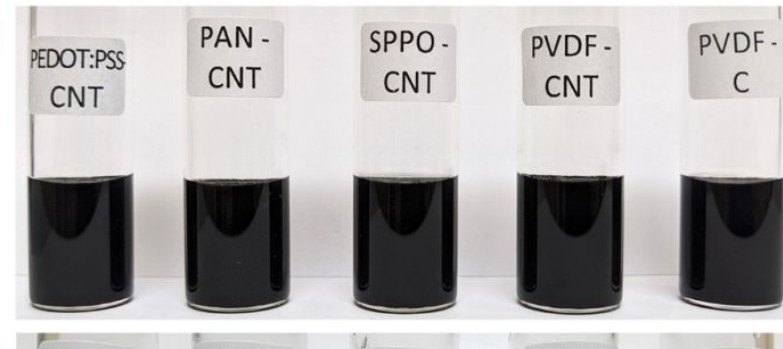

(b)
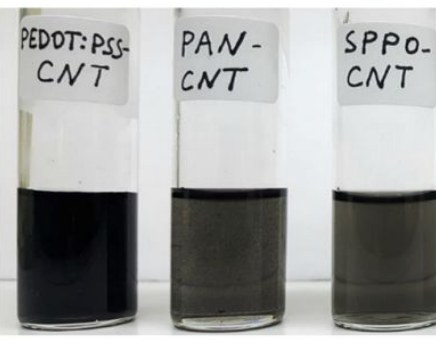

SPPO- PVDF-

CNT

CNT

PVDF-

$C$

(c)

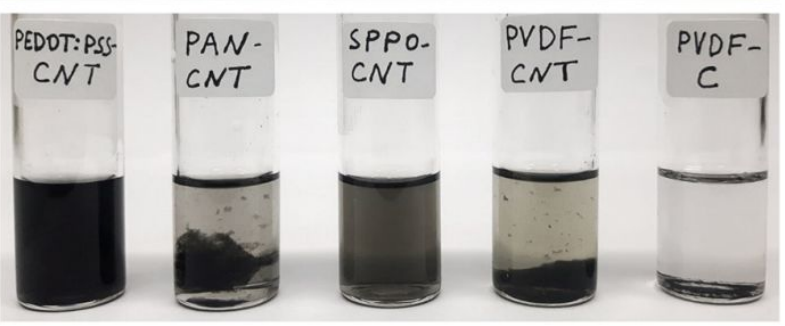

Figure S3. Dispersion stability of SWCNT and Super-C65 carbon black in the polymer solutions: SWCNT in PEDOT:PSS solution $\left(\mathrm{H}_{2} \mathrm{O}\right)$, SWCNT in PAN solution (DMSO), SWCNT in SPPO solution $\left(\mathrm{H}_{2} \mathrm{O}-\mathrm{EtOH}\right)$, SWCNT in PVDF solution (NMP), and carbon black in PVDF solution (NMP); (a) initial dispersions ( $1 \mathrm{mg} \mathrm{mL}^{-1} \mathrm{SWCNT} /$ carbon black, $20 \mathrm{mg} \mathrm{mL}^{-1}$ polymer), (b) 1 hour after the dilution of initial dispersions with the solvent in 100 times, (c) 3 days after the dilution of initial dispersions with the solvent in 100 times. 

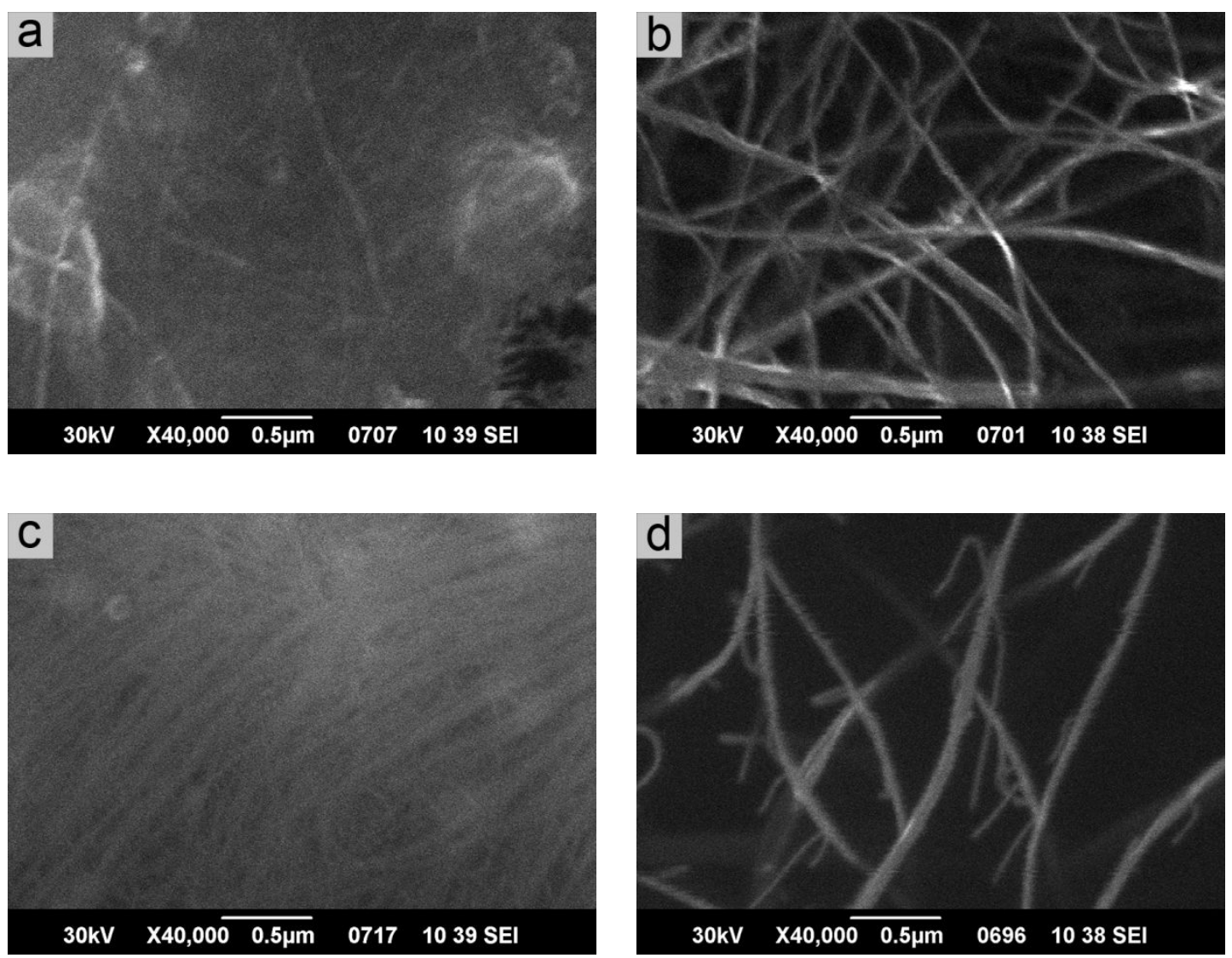

Figure S4. SEM images of a - PEDOT:PSS-SWCNT, b - PAN-SWCNT, $\mathbf{c}-$ SPPO-SWCNT, d PVDF-SWCNT films.

Table S1. Surface energy parameters for the polymer binder films and aluminum foil current collector.

\begin{tabular}{|c|c|c|c|c|c|}
\hline Sample & $\begin{array}{c}\text { Surface } \\
\text { energy, } \\
\gamma_{s}, \mathrm{~mJ} \mathrm{~m}^{-2}\end{array}$ & $\begin{array}{c}\text { Polar } \\
\text { component, } \\
\gamma_{s}^{p}, \mathrm{~mJ} \mathrm{~m}^{-2}\end{array}$ & $\begin{array}{l}\text { Dispersive } \\
\text { component, } \\
\gamma_{s}^{d}, \mathrm{~mJ} \mathrm{~m}^{-2}\end{array}$ & $\begin{array}{l}\text { Surface } \\
\text { polarity, } \\
\chi=\gamma_{s}^{p} / \gamma_{s}\end{array}$ & Reference \\
\hline \multirow{3}{*}{ PVDF } & 30 & 7 & 23 & 0.23 & (1) \\
\hline & 34 & 3 & 31 & 0.08 & (2) \\
\hline & 36 & 3 & 33 & 0.08 & (3) \\
\hline \multirow{3}{*}{ PAN } & 41 & 10 & 31 & 0.24 & (4) \\
\hline & 51 & 10 & 41 & 0.20 & (5) \\
\hline & 54 & 14 & 40 & 0.25 & (6) \\
\hline SPPO & $54 *$ & N/A & N/A & N/A & N/A \\
\hline
\end{tabular}




\begin{tabular}{cccccc}
\hline & 52 & 30 & 22 & 0.58 & $(7)$ \\
PEDOT:PSS & 72 & 46 & 25 & 0.65 & $(8)$ \\
& 73 & 46 & 27 & 0.63 & $(9)$ \\
Al-foil & 34 & 7 & 27 & 0.21 & $(10)$ \\
& 37 & N/A & N/A & N/A & $(11)$ \\
\hline
\end{tabular}

*Estimated from molecular structure using ACD/ChemSketch software

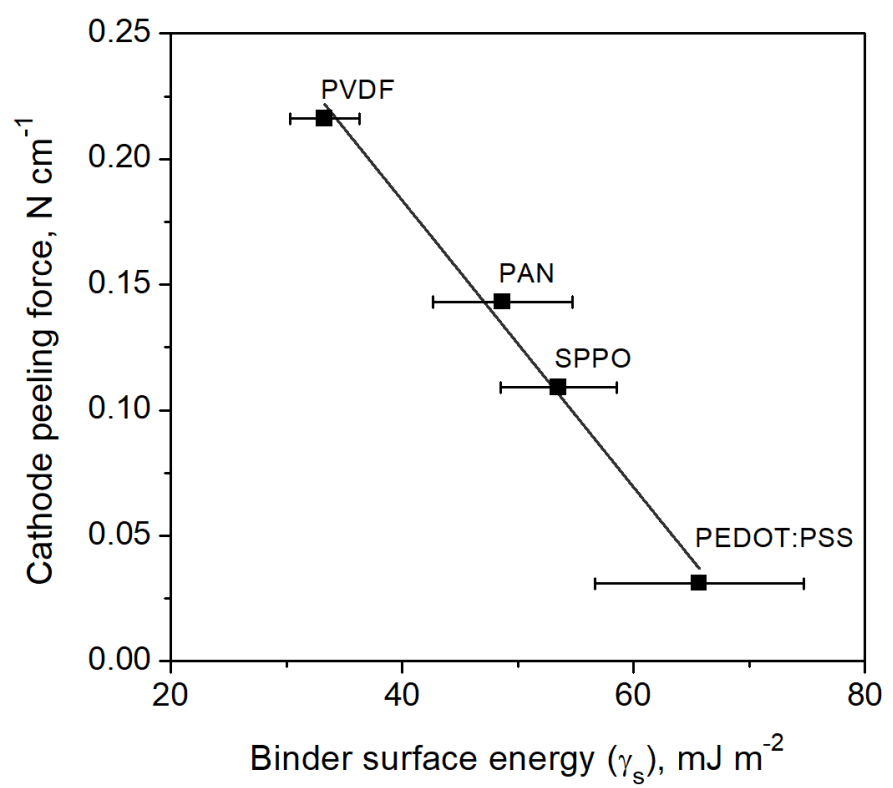

Figure S5. Effect of the surface free energy of the polymer binder on the peeling force of the $\mathrm{LiFePO}_{4}$ composite cathode. 
Table S2. Peeling force values of the composite cathodes depending on the amount of PVDF binder.

\begin{tabular}{|c|c|c|c|}
\hline $\begin{array}{c}\text { Content of LFP, } \\
\text { wt. } \%\end{array}$ & $\begin{array}{c}\text { Content of carbon, } \\
\text { wt. } \%\end{array}$ & $\begin{array}{c}\text { Content of PVDF, } \\
\text { wt. } \%\end{array}$ & $\begin{array}{l}\text { Peeling force, } \\
\qquad \mathrm{N} \mathrm{cm}^{-1}\end{array}$ \\
\hline \multirow[t]{2}{*}{95} & 2.5 & 2.5 & 0.03 \\
\hline & (carbon black) & & \\
\hline \multirow[t]{2}{*}{95} & 0.25 & 4.75 & 0.21 \\
\hline & (carbon black) & & \\
\hline \multirow[t]{2}{*}{95} & 0.25 & 4.75 & 0.22 \\
\hline & (SWCNT) & & \\
\hline \multirow[t]{2}{*}{80} & 10 & 10 & 0.29 \\
\hline & (carbon black) & & \\
\hline
\end{tabular}

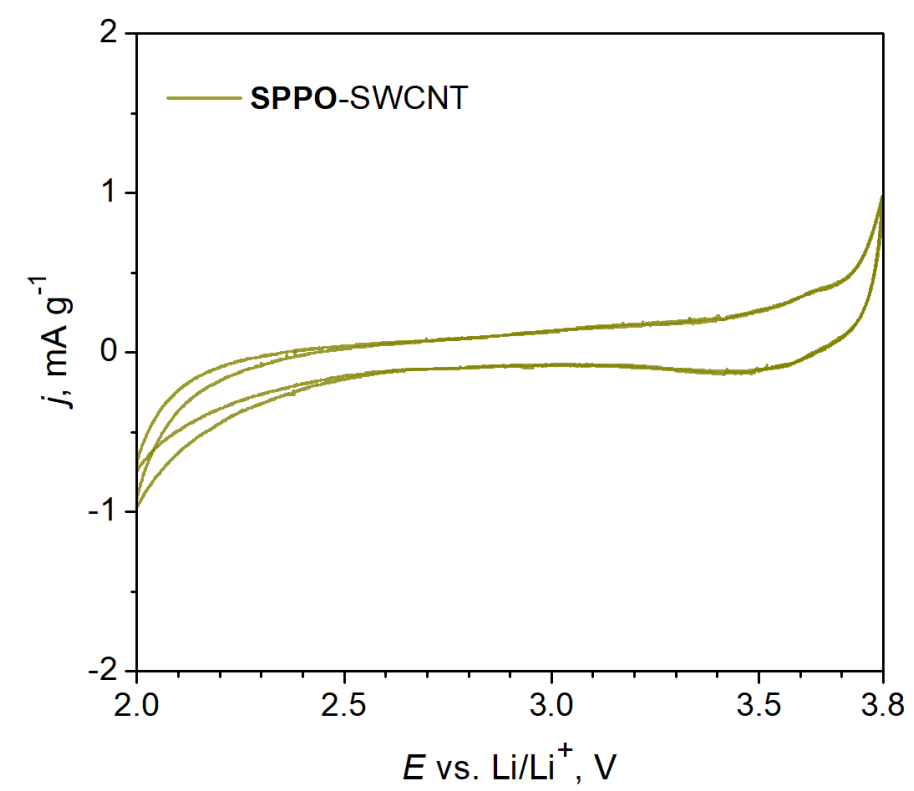

Figure S6. Cyclic voltammograms of the SPPO-SWCNT film $\left(50 \mu \mathrm{V} \mathrm{s}^{-1}\right)$.

\section{REFERENCES}

(1) Wu, S. Calculation of Interfacial Tension in Polymer Systems. J. Polym. Sci., Part C: Polym. Symp. 1971, 34 (1), 19-30. DOI: 10.1002/polc.5070340105 
(2) Boulangé-Petermann, L.; Debacq, C.; Poiret, P.; Cromières, B. Effect of the Physical Chemistry of Polymeric Coating Surfaces on Fouling and Cleanability with Particular Reference to the Food Industry. In Contact Angle, Wettability and Adhesion; Vol. 3; Mittal, K. L., Ed.; CRC Press, 2003; pp 501-519. DOI: 10.1201/9789047403326-33

(3) Dalal, E. N. Calculation of Solid Surface Tensions. Langmuir 1987, 3 (6), 1009-1015. DOI: 10.1021/la00078a023

(4) Yushkin, A. A.; Efimov, M. N.; Vasilev, A. A.; Bogdanova, Yu. G.; Dolzhikova, V. D.; Karpacheva, G. P.; Volkov, A. V. Modification of Polyacrylonitrile Membranes by Incoherent IR Radiation. Pet. Chem. 2017, 57 (4), 341-346. DOI: 10.1134/S0965544117040089

(5) Xu, Y. C.; Wang, Z. X.; Cheng, X. Q.; Xiao, Y. C.; Shao, L. Positively Charged Nanofiltration Membranes via Economically Mussel-Substance-Simulated Co-Deposition for Textile Wastewater Treatment. Chem. Eng. Journal 2016, 303, 555-564. DOI: 10.1016/j.cej.2016.06.024

(6) Lim, J. W.; Lee, J.-M.; Yun, S.-M.; Park, B.-J.; Lee, Y.-S. Hydrophilic Modification of Polyacrylonitrile Membranes by Oxyfluorination. J. Ind. Eng. Chem. 2009, 15 (6), 876-882. DOI: 10.1016/j.jiec.2009.09.016

(7) Lee, H. J.; Park, T. H.; Choi, J. H.; Song, E. H.; Shin, S. J.; Kim, H.; Choi, K. C.; Park, Y. W.; Ju, B.-K. Negative Mold Transfer Patterned Conductive Polymer Electrode for Flexible Organic LightEmitting Diodes. Org. Electron. 2013, 14 (1), 416-422. DOI: 10.1016/j.orgel.2012.11.015

(8) Petrosino, M.; Rubino, A. The Effect of the PEDOT:PSS Surface Energy on the Interface Potential Barrier. Synth. Met. 2012, 161 (23), 2714-2717. DOI: 10.1016/j.synthmet.2011.10.006

(9) Vacca, P.; Petrosino, M.; Miscioscia, R.; Nenna, G.; Minarini, C.; Della Sala, D.; Rubino, A. Poly(3,4-Ethylenedioxythiophene):Poly(4-Styrenesulfonate) Ratio: Structural, Physical and Hole Injection Properties in Organic Light Emitting Diodes. Thin Solid Films 2008, 516 (12), 4232-4237. DOI: $10.1016 /$ j.tsf.2007.12.143

(10) Vengatesh, P.; Kulandainathan, M. A. Hierarchically Ordered Self-Lubricating Superhydrophobic Anodized Aluminum Surfaces with Enhanced Corrosion Resistance. ACS Appl. Mater. Interfaces 2015, 7 (3), 1516-1526. DOI: 10.1021/am506568v

(11) Li, J.; Armstrong, B.; Kiggans, J.; Daniel, C.; Wood, D. Influence of Al Foil Surface Energy and Mixing Method on $\mathrm{LiFePO}_{4}$ Cathode Fabrication. Meet. Abstr. 2011. DOI: 10.1149/MA2011$02 / 7 / 405$ 
(12) Hansen, M. H.; Finlayson, M. F.; Goins, D. The Role of Corona Discharge Treatment in Improving Polyethylene-Aluminum Adhesion: An Acid-Base Perspective. TAPPI J. 1993, 76 (2), 171-177 